\title{
A evolução das relações pais- escola, particularmente dos pais de alunos em dificuldade de aprendizagem
}

Parents- school's evolution relation, particularly
learning difficulty students' parents L'évolution des relationes parents-école,
particulièrement des parents d'éleves en difficulté
d'apprentissage

Caroline DALLAIRE

\section{RESUMO}

Este depoimento apresenta uma situação real de vida profissional vivida pela autora em que a mesma procura o apoio da família de um aluno em situação de dificuldade de aprendizagem da leitura e da escrita em francês.

Palavras- chave: dificuldade, aprendizagem, leitura, escrita, escola, pais.

\section{ABSTRACT}

This report presents a real professional life situation by the author. Inside this, the author supports one reading and writing French difficulty tearning situation student family.

Index terms: difficulty, learning, reading, writing, school, parents.

\section{RÉSUMÉ}

Cette temoignage présente une situation réelle de vie professionnelle vécue par l'auteure où la même recherche d'aider une famille d'un élève en situation de difficulté d'apprentissage de la lecture et de l'écriture en Français.

Mots cles: difficulté, apprentissage, lecture, écriture, école, parents. 
Cette année, un jeune garçon qui est en quatrième année est arrivé à l'école où je travaille. Il allait auparavant dans une école privée de la région, mais les parents n'étaient pas satisfaits des services offerts. À son arrivée à l'école, on constate rapidement qu'il est en grande difficulté en lecture et en écriture. On fait alors une évaluation avec l'accord des parents, car nous soupçonnons un trouble spécifique de la lecture et de l'écriture. Il s'avère après notre évaluation et celle de l'orthophoniste que cet enfant a une dyslexie et une dysorthographie sévères. Les parents sont donc informés des résultats. Évidemment, c'est un choc et les parents, surtout la mère, le prennent très mal.

Après quelques discussions avec la mère, je comprends qu'ils lont décidé de le changer d'école, car à son ancienne école, les professeurs disaient sans cesse aux parents que leur enfant était en difficulté et échouerait si la situation ne s'améliorait pas. Comme dans les écoles privées il n'y a pas d'orthopédagogues, aucune ressource n'était disponible pour lui. Alors, ils ont décidé de l'envoyer dans une école publique croyant que ce serait plus facile pour lui au niveau académique, mais malheureusement, cela ne s'est pas produit ainsi. Ayant beaucoup de difficulté à accepter le diagnostic de son fils, la mère met le tout sur la faute de l'école.

Le lien est donc très difficile à établir avec elle. Cêtte dernière en veut à l'enseignante de son fils, car elle dit qu'elle est toujours sur son dos, qu'elle ne fait rien pour l'aider et qu'elle est toujours négative dans ses commentaires. L'enfant sent alors la tension entre les deux parties et en profite. Pour garder sa motivation et avoir le goût d'apprendre, l'enfant doit sentir que ses parents accordent de l'importance aux apprentissages qu'il réalise à l'extérieur du contexte familial.

Avec ma direction, nous avons donc invité les parents et l'enseignante à une rencontre explicative. J'ai alors expliqué plus en détails aux parents et à l'enseignante ce qu'était la dyslexie et la dysorthographie. 
Nous avons ensuite discuté de l'aide que j'apporterais à leur fils et des adaptations qui pourraient se faire en classe.

Cette rencontre a eu pour effet de faire comprendre aux parents que personne n'est responsable de ce trouble. Tout d'abord, je pense que quand on comprend une situation, on est plus apte à l'accepter. La mère a donc pris conscience lors de cette réunion que son support pouvait aider son fils et faire une différence dans sa réussite scolaire. Elle a même demandé par la suite ce qui lui était possible de faire à la maison pour lui venir en aide. D'ailleurs, les résultats d'une étude menée par Deslandes auprès de 1227 parents d'élèves du primaire indiquent que quel que soit la scolarité du parent, la taille de la famille, le sexe de l'enfant et son niveau scolaire et que le parent travaille ou non à l'extérieur, plus les parents ont l'impression d'avoir un impact sur le rendement scolaire de l'enfant et de posséder les ressources requises (sentiment de compétence parentale), plús ils s'impliquent à la maison. Elle mentionne également lors de cette même étude que des relations positives entre les parents et l'école contribuent aussi à soutenir les parents dans leur rôle parental et à développer leur sentiment de compétence parentale dans l'aide à apporter à leur enfant. De leur côté, les enseignantes en viennent à développer une attitude plus positive à l'égard des parents comme éducateurs. ${ }^{1}$

Je pense que cette réunion a servi non seulement à établir un lien de confiance entre l'école et la famille, mais aussi à ce que chacun comprenne l'importance de travailler ensemble dans l'intérêt de l'enfant. La confiance mutuelle entre les parents et les enseignants apparaît comme une composante vitale à des relations collaboratives. Depuis cette réunion, un système de communication simple, efficace et choisi par les parents et l'enseignante a été mis en place afin de suivre le cheminement de l'élève. De cette façon, l'enfant voit maintenant qu'il y a un lien entre l'école et la

${ }^{1}$ DESLANDES, R.. Collaboration école-famille: défis sociaux et scolaires. Options CSQ Hors Série, n.1. p. 145$167,2006$. 
maison et je pense qu'il est important pour l'enfant qu'il y ait ce lien, afin de lui permettre de maximiser ses chances de réussite. De plus, pendant cette rencontre, les parents ont pu constater que l'enseignante était aussi un peu démunie face à cette situation, car c'était la toute première fois qu'elle accueillait dans sa classe un élève avec cette problématique. Ils ont donc pu constater qu'ils n'étaient pas les seuls à se sentir vulnérables face à cette situation. Je pense donc qu'il est important que chacun soit transparent dans une situation comme celle-là, car cela facilite le lien de confiance entre les parties.

Après cette réunion, j'ai commencé à travailler en étroite collaboration avec l'enseignante de cet élèvé. Je lui ai fourni beaucoup de documentation sur la dyslexie et la dysorthographie ainsi que plusfeurs conseils pratiques et concrets qui peuvent être facilement utilisés en classe. L'enseignante m'a alors confié être rassurée face à cette situation êt a, à partir de ce moment, été prête à s'investir davantage avec l'élève èt à changer ses façons de faire. D'ailleurs, selon Paolo Freire, il est nécessaire d'investir dans le changement, en particulier dans l'enseignement, car rien dans ce monde n'est statique ni permanent et une partie de la fonction d'enseignante est de s'ouvrir aux nouveaux horizons, d'être critique de soimême et de toujours user de sa créativité pour que lenseignementapprentissage apporte quelque chose de satisfaisant aux deux parties (élève et enseignant). ${ }^{2}$

Je pense aussi que le fait d'être mieux informés par rapport à cette problématique a permis à l'enseignante et aux parents de regagner confiance en eux et ils ont commencé à voir cela comme un défi plutôt que comme une lourde tâche. En début d'année, l'enseignante me confiait qu'elle trouvait difficile d'avoir un élève en si grande difficulté dans sa classe et qu'elle croyait qu'étant donné ses difficultés, elle pensait qu'il serait

\footnotetext{
${ }^{2}$ FREIRE, P.. Professeur et résilience: 1"importance de la créativité et de flexibilité. (http://fr.shvoong.com/socialsciences/education/1876534-professeur-et-r\%C3\%A9silience-importance-la/), Mar., 2008.
} 
beaucoup plus à sa place dans une classe spéciale, perception qu'elle transmettait sans nécessairement le vouloir à l'enfant et à ses parents. Je lui expliquais alors que malgré sa difficulté à lire et à écrire, c'était un enfant extrêmement brillant qui pouvait réussir si quelques moyens étaient mis en place pour pallier à ses difficultés. J'ai alors pu constater que parfois seul le fait de se retrouver devant l'inconnu peut complètement modifier la façon de penser d'une personne. Dans ce cas, l'enseignante avait jugé l'enfant trop vite, car elle n'était pas familière avec ce trouble. Je crois donc qu'il est de notre rôle en tant que spécialiste des troubles d'apprentissage d'ouvrir les enseignants de classe ordinaire et par le fait même les parents aux difficultés d'apprentissage et à l'intégration, car on sait que l'inconnu fait peur êt je crois que cette peur a un grand rôle à jouer dans une bonne intégration des élèves en difficulté d'apprentissage. Il est, à mon avis, important que tous comprenne qu'avec un trouble d'apprentissage comme celui-ci, l'intégration est la meilleure solution pour cet enfant, car cela lui permettra une progression optimale. Comme le disait Jean Biarnès lorsqu'il parlait de réintroduire la complexité, il faut permettre à l'élève de réaliser ses rêves en l'accompagnant et en l'aidant à traverser chaque étape, ce qui selon moi, est plus facile à réaliser dans une classe ordinaire. ${ }^{3}$

La nouvelle attitude d'ouverture de l'enseignante face au jeune a eu un impact majeur d'abord sur l'élève, mais également sur ses parents. Comme ils sentent maintenant que leur enfant est bien supporté, ils ont confiance en l'enseignante et s'impliquent davantage. En 2002, Perrenoud écrivait d'ailleurs qu'une plus grande confiance à l'égard des enseignants passe nécessairement par la relation que l'enseignant établit avec l'enfant. ${ }^{4}$

Pour terminer, je pense que les relations parents-école sont comme toutes les relations de notre vie. Tout d'abord, elles prennent du temps à se

\footnotetext{
${ }^{3}$ BIARNÈS, J.. Université, diversité, sujet dans l'espace pédagogique. Paris: L'Harmattan. P. 13-32, 1999. ${ }^{4}$ PERRENOUD, P.. Quelques conditions d'un partenariat durable entre les parents et les enseignants. In : http://webcache.googleusercontent.com/search?q=cache:glEmGuPUCLMJ:www.unige.ch/fapse/SSE/teachers/perr enoud/php_main/familles.html+quelques+condition+perrenoud\&cd=2\&hl=en\&ct=clnk\&gl=ca, Mars., 2008.
} 
bâtir et chacun se doit de respecter le niveau d'implication de l'autre. Ensuite, pour être cordiales et surtout pour durer, elles nécessitent un travail de longue haleine et chacune des parties en cause doit se faire confiance, se respecter et tenir compte des compétences et des attentes de chacun.

\section{Autora:}

Caroline Dallaire

Ortopedagoga que atua na região do Quebec, Canadá.

Contato: caroline.dallaire@uqac.ca

Texto recebido em maio de 2009.

Texto aprovado para publicação em julho de 2009.

\section{Como citar este texto:}

DALLAIRE, C.. L'évolution des relationes parents-école, particulièrement des parents d'éleves en difficulté d'apprentissage. Revista Acolhendo a Alfabetização nos Países de Língua Portuguesa, Brasil, São Pảulo, volume 1, $\mathrm{n}^{\circ}$. 10, pp. 151 - 156, Mar. 2011. Disponíyel-em: $\langle$ http://www.acoalfaplp.net>. 